Conclusion The findings indicate that telemedicine in CF is feasible but the uptake amongst people with CF may be challenging. This is probably not surprising since adherence to treatment is often poor. Nevertheless telemedicine has the potential to play an important role in the early detection of pulmonary exacerbations and further studies are required.

\section{P279 THE FEMALE DISADVANTAGE IN UK CF REGISTRY DATA 2008-2013}

${ }^{1} \mathrm{~S}$ Hippolyte, ${ }^{2} \mathrm{R}$ Keogh, ${ }^{3} \mathrm{~S}$ MacNeill, ${ }^{1} \mathrm{~N}$ Simmonds, ${ }^{4} \mathrm{U}$ Griesenbach. ${ }^{1}$ Royal Brompton and Harefield NHS Trust, London, UK; ' ${ }^{2}$ London School of Hygiene and Tropical Medicine, London, UK; ${ }^{3}$ School of Social and Community Medicine, Bristol, UK; ${ }^{4}$ Gene Therapy Group, NHLI, Imperial College, London, UK

\subsection{6/thoraxjnl-2015-207770.415}

Introduction and objectives The UK Cystic Fibrosis (CF) registry has been in its current form since 2006 offering annual review data comprising of detailed demographic and clinical information on $99 \%$ of the UK CF population (>10,000 individuals). Whilst widely accepted that $\mathrm{FEV}_{1}$ and BMI are well-validated predictors of disease severity and outcome, the role of gender in CF remains debated. In some studies Pseudomonas aeruginosa (PsA) infection occurred earlier in females with a resulting deterioration in $\mathrm{FEV}_{1}$. Here, we use CF registry data (2008-2013) to assess whether a female disadvantage in $\mathrm{FEV}_{1}$ or BMI exists in the UK population and whether PsA status differs by gender. This is the most complete UK CF registry gender-based analysis to date.

Methods and results Cross-sectional analysis of data from 2010 and 2013 supported decreased female survival (decreasing female prevalence with sequential age groups; $2013 \mathrm{p}=$ 0.0001). It also highlighted lower BMI percentiles and more underweight (BMI <19) individuals amongst females $(21.9 \%$; males 13.6\%: $\mathrm{p}=<0.0001$ ), even when adjusted for lung function.

Females had worse lung function compared to males, particularly in adolescence; (females: mean $\mathrm{FEV}_{1} 71.3 \%$ at $16-19 \mathrm{yrs}$ (CI 69.2-73.4), males: 78.9\% (CI 76.9-81.0); $\mathrm{p}<0.0001$ ).

Females had higher absolute rates $(57.1 \%$ on any intravenous antibiotics; $44.8 \%$ males) and greater total duration of intravenous antibiotic use across all adult age groups $(\mathrm{p}<)$.

Females had higher rates of CF-related diabetes from 16-29 years (females $28.2 \%$, males $17.7 \%$ : $\mathrm{p}<0.005$ ), itself independently associated with worse prognosis.

On full analysis from 2008-2013 the age at which chronic PsA was first reported occurred earlier in females (mean 15.5 yrs 95\% CI 14.9-16.1) than males (16.7 yrs; 95\% CI 16.1-17.3) $\mathrm{p}=0.01$.

Conclusions Disease severity appears worse in CF females compared to males on cross-sectional analysis of data from 2010 and 2013. Females have earlier PsA infection and lower BMI, both of which are individually associated with worse outcomes and increased intravenous antibiotic use. Females also have reduced lung function, and receive more treatment. These data suggest a persistent and measurable gender difference in the UK CF population which we aim to explore more closely in longitudinal analysis.

\section{P280 A SINGLE CENTRE EXPERIENCE OF SPONTANEOUS CLEARANCE OF MYCOBACTERIUM ABSCESSUS IN CYSTIC FIBROSIS PATIENTS}

HD Green, PJ Barry, R Bright-Thomas, AM Brennan, AK Webb, R Lord, A Horsley, AM Jones. University Hospital of South Manchester, Manchester, UK

10.1136/thoraxjnl-2015-207770.416

Introduction The Mycobacterium abscessus complex is an emerging group of pathogens, which pose significant management challenges in CF. Current guidelines specify treatment is indicated in patients with repeated sputum culture positivity alongside radiological or clinical deterioration. However, identifying NTM as the cause of deterioration in the polymicrobial CF lung is challenging. Additionally, M. abscessus complex isolates are usually multi-resistant, requiring lengthy and complex treatment regimens. Whether to treat patients based on culture results alone is contentious and approaches differ between centres. Here we analyse our experience of $M$. abscessus at a large UK adult CF centre.

Methods All patients with 1 or more positive sputum culture for M. abscessus since 2010, and minimum of 1 mycobacterial culture and 1 year of follow-up since first positivity were included. Anti-mycobacterial treatment and culture results following first positivity were recorded. M. abscessus eradication was defined as 4 consecutive negative cultures spanning at least 1 year.

Results 21 patients were included. Of these, 6 (29\%) have received/are receiving, anti-mycobacterial therapy based on clinician diagnosis of M. abscessus pulmonary disease. All 6 currently remain culture positive. Of the 15 remaining patients, 6 are consistently culture positive (duration 12 months - 5 years), but do not have evidence of NTM pulmonary disease. Spontaneous clearance of $M$. abscessus from sputum has occurred in 7 patients (Table 1$)$. Of these, $5(71 \%)$ had $\geq 3$ positive cultures including 1 patient with 5 positive samples spread over 2 years and 1 patient with 5 positive samples spread over 9 months. In 2 patients infection status cannot yet be confirmed as these patients have $\leq 4$ mycobacterial culture results following their initial positive result.

Abstract P280 Table 1 Mycobacterium abscessus Sputum Culture and Treatment Status of Patients Attending Manchester Adult Cystic Fibrosis Centre

\begin{tabular}{|c|c|}
\hline Persistently culture positive with history of treatment, $n(\%)$ & $6(31.6)$ \\
\hline Persistently culture positive but never treated, $\mathrm{n}(\%)$ & $6(31.6)$ \\
\hline Spontaneous clearance from sputum, $\mathrm{n}(\%)$ & $7(36.8)$ \\
\hline Total number of patients & 19 \\
\hline
\end{tabular}

Conclusion Patients may spontaneously clear M. abscessus from their sputum, even with a history of multiple positive cultures over many months. If patients are treated on culture results alone there is a risk of initiating potentially unnecessary, lengthy and poorly tolerated treatment. Our results suggest that adhering to clinical guidelines of recognising clinical deterioration secondary to M. abscessus remains paramount before commencing treatment and assessment of treatment success without control data may be very misleading. 